\title{
Experiência inicial com a ceratoprótese de Dohlman-Doane: relato de casos
}

\author{
Experience with Dohlman-Doane keratoprosthesis: case reports
}

\author{
Andressa Prestes Stolz ${ }^{1}$ \\ Sérgłio Kwitko ${ }^{2}$ \\ Melissa Manfroi Dal Pizzol \\ Diane Marinho ${ }^{4}$ \\ Samuel Rymer ${ }^{5}$
}

\begin{tabular}{|l|}
\hline RESUMO \\
\hline Objetivo: Relatar a evolução de 9 olhos (8 pacientes) submetidos ao \\
implante da ceratoprótese de Dohlman-Doane tipo 1, com tempo médio de \\
seguimento de 11,2 meses (2a 25 meses). Métodos: Trabalho retrospectivo, \\
não comparativo, intervencionista de uma série de casos. Quanto à doença \\
de base, em 4 olhos foram queimadura alcalina, 3 falência de múltiplos \\
transplantes de córnea, 1 síndrome de Stevens-Johnson e 1 queimadura \\
térmica. A acuidade visual pré-operatória era de movimento de mãos ou \\
pior em todos os olhos. Três olhos apresentavam glaucoma pré-operató- \\
rio, sendo submetidos a implante valvulado de Ahmed. Resultados: A \\
acuidade visual com correção pós-operatória foi melhor ou igual a 20/100 \\
em 88,9\% dos olhos e melhor ou igual a 20/40 em 44,4\% dos olhos. Como \\
intercorrências pós-operatórias, 3 olhos apresentaram opacidade de cáp- \\
sula posterior tratada com capsulotomia com YAGlaser; 3 olhos membrana \\
retroprotética de fibrina tratada com injeção tPA ou corticoterapia; 2 olhos \\
glaucoma tratado com medicação; 1 olho “melting" corneano tratado com \\
a troca do botão e 1 olho endoftalmite fúngica tratada com transplante a \\
quente, vitrectomia anterior, explante da ceratoprótese da LIO e tratamen- \\
to antifúngico. Conclusão: A ceratoprótese de Dohlman-Doaneé umaboa \\
opção para casos graves de cegueira corneana, em que o transplante pe- \\
netrante de córnea não apresenta bom prognóstico. Sua principal vanta- \\
gem é não necessitar imunossupressão sistêmica. Casos de queimadura \\
alcalina e de alta chance de rejeição apresentaram os melhores resultados. \\
\hline
\end{tabular}

Descritores: Próteses e implantes; Ceratoplastia penetrante; Implante de prótese/métodos; Doenças da córnea/cirurgia; Cegueira; Relatos de casos [Tipo de publicação]

\section{INTRODUCÃO}

Em casos de múltiplos transplantes de córnea, a cada novo enxerto corneano aumentam os riscos de rejeição e de complicações como glaucoma e edema macular, enquanto o tempo médio de sobrevivência do enxerto diminui ${ }^{(1)}$.

A ceratoprótese de Dohlman-Doane ou "Boston keratoprosthesis" (KPro) tem sido uma importante alternativa nos casos de cegueira corneana para os quais o transplante penetrante de córnea não apresenta bom prognóstico ${ }^{(2-3)}$.

Dois tipos de KPro de Dohlman-Doane foram desenvolvidos, uma para pacientes com relativa boa hidratação da superfície ocular e fechamento palpebral (tipo 1) e outra para casos de grave olho seco, usada trans-palpebralmente (tipo 2). Apesar dos resultados mostrarem-se favoráveis, existem diversas complicações que ameaçam o prognóstico a longo prazo desses implantes. Isto inclui extrusão da prótese, formação de membrana retro-protética, 
necrose corneana, crescimento epitelial intracameral, glaucoma, descolamento de retina e endoftalmite ${ }^{(4)}$.

O objetivo deste trabalho é relatar os casos de 9 olhos de 8 pacientes submetidos ao implante da ceratoprótese de DohlmanDoane tipo 1.

\section{MÉTODOS}

Trabalho retrospectivo, não comparativo, intervencionista de uma série de casos submetidos ao implante de KPro de Dohlman-Doane tipo 1.

Foram analisados 9 olhos de 8 pacientes com média de idade de 29,7 anos (13-62 anos). Quanto às patologias de base, 4 olhos de 3 pacientes haviam sofrido queimadura alcalina grave, 3 olhos apresentavam falência de múltiplos transplantes de córnea prévios (ceratocone, ceratopatia bolhosa do afácico e ceratite por Acanthamoeba), 1 olho com síndrome de Stevens-Johnson (SSJ) grave e 1 olho com queimadura térmica grave. Todos os olhos apresentavam acuidade visual (AV) pré-operatória igual ou menor que movimento de mãos (MM) e tiveram diversas cirurgias realizadas anteriormente ao implante da ceratoprótese. A tabela 1 demonstra as características dos casos operados.

Antibioticoprofilaxia tópica com fluoroquinolona de $4^{\underline{a}}$ geração (gatifloxacina) foi utilizada em todos os casos. Todas as

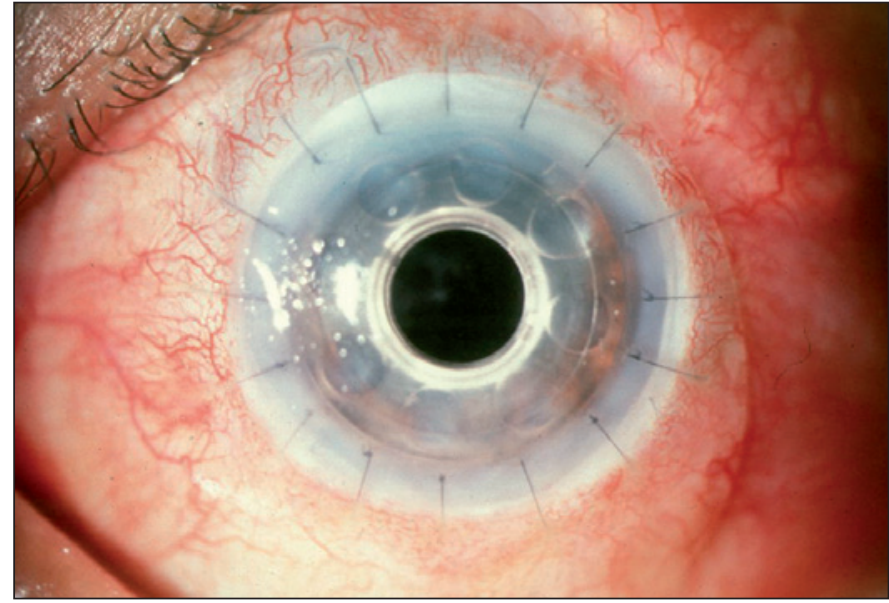

Figura 1 - Aspecto nos 18 meses de pós-operatório do caso 2

cirurgias foram realizadas pelo mesmo cirurgião (SK). A técnica cirúrgica encontra-se descrita na literatura ${ }^{(2,5-6)}$. Em 7 olhos foram utilizadas ceratopróteses para pseudofacia e em 2 olhos para afacia. Em 4 olhos foi realizada facectomia com implante de lente intra-ocular (LIO) no saco capsular no mesmo tempo cirúrgico, 4 olhos já eram pseudodfácicos e as lentes foram mantidas. Dois olhos eram e foram mantidos afácicos. Três olhos apresentavam glaucoma no pré-operatório, sendo submetidos ao implante

\begin{tabular}{|c|c|c|c|c|c|c|c|}
\hline Olhos & $\begin{array}{l}\text { Idade } \\
\text { (anos) }\end{array}$ & Sexo & $\begin{array}{l}\text { Patologia de } \\
\text { base }\end{array}$ & Olho & Cirurgias prévias & $\begin{array}{c}\text { PIO } \\
\text { pré-operatória }\end{array}$ & $\begin{array}{c}\text { AV } \\
\text { pré-operatória }\end{array}$ \\
\hline 1 & 16 & M & Queimadura alcalina & OE & $\begin{array}{c}3 \text { Tx MA } \\
2 \text { Tx limbo (pai) }\end{array}$ & Normal & $\mathrm{MM}$ \\
\hline 2 & 31 & M & Queimadura alcalina & OE & $\begin{array}{c}1 \text { Tx limbo (irmão) } \\
1 \text { Tx Co } \\
1 \text { Tx MA }\end{array}$ & Aumentada & MM \\
\hline 3 & 62 & $\mathrm{~F}$ & $\begin{array}{l}\text { Síndrome de } \\
\text { Stevens-Johnson }\end{array}$ & OD & $\begin{array}{c}3 \text { Tx Co } \\
1 \text { tríplice } \\
1 \text { Tx limbo cadáver } \\
\text { Enxerto gl. salivar }\end{array}$ & Normal & MM \\
\hline 4 & 51 & $\mathrm{~F}$ & $\begin{array}{c}\text { Ceratocone } \\
\text { (falência Tx prévios) }\end{array}$ & OE & $\begin{array}{l}1 \mathrm{Tx} \text { Co } \\
1 \text { tríplice }\end{array}$ & Normal & MM \\
\hline 5 & 16 & M & Queimadura alcalina & OD & $\begin{array}{c}1 \mathrm{Tx} M A \\
1 \mathrm{Tx} \operatorname{limbo}(\text { pai) }\end{array}$ & Normal & MM \\
\hline 6 & 22 & M & $\begin{array}{l}\text { Ceratite por Acanthamoeba } \\
\text { (falência Tx prévios) }\end{array}$ & OE & $\begin{array}{c}1 \text { recobrimento conjuntival } \\
1 \text { Tx Co a quente } \\
1 \text { implante Ahmed }\end{array}$ & Aumentada & MM \\
\hline 7 & 13 & M & Queimadura alcalina & OD & $\begin{array}{l}4 \text { reconstr. sup. MA } \\
1 \text { Tx limbo (mãe) } \\
1 \text { Tx limbo cadáver }\end{array}$ & Normal & MM \\
\hline 8 & 36 & M & Queimadura térmica & OD & $2 \mathrm{Tx}$ Co & Normal & $C D$ \\
\hline 9 & 20 & $\mathrm{~F}$ & $\begin{array}{l}\text { Ceratopatia bolhosa } \\
\text { pós facectomia } \\
\text { (falência Tx prévios) }\end{array}$ & OE & $\begin{array}{c}1 \text { facoaspiração } \\
2 \text { trabeculectomias } \\
3 \mathrm{Tx} \text { Co } \\
1 \text { retinopexia }\end{array}$ & Aumentada & PL c/ cores \\
\hline \multicolumn{8}{|c|}{$\begin{array}{l}\mathrm{PIO}=\text { pressão intra-ocular; } \mathrm{AV}=\text { acuidade visual; } \mathrm{M}=\text { masculino; } \mathrm{F}=\text { feminino; } \mathrm{OD}=\text { olho direito; } \mathrm{OE}=\text { olho esquerdo; } \mathrm{Tx}=\text { transplante; } \mathrm{Co}=\text { córnea; } \mathrm{MA}=\text { membrana amniótica; } \\
\text { tríplice= transplante penetrante de córnea com facectomia e implante de lente intra-ocular; } \mathrm{MM}=\text { movimento de mãos; } \mathrm{CD}=\mathrm{conta} \text { dedos; } \mathrm{PL} \mathrm{c} / \mathrm{cores=}=\mathrm{percepção} \mathrm{luminosa} \\
\text { com distinção de cores }\end{array}$} \\
\hline
\end{tabular}


valvulado de Ahmed (um previamente e dois simultaneamente à cirurgia da KPro). No final do procedimento todos os olhos receberam injeção de $4 \mu \mathrm{g}$ de dexametasona intracameral.

No pós-operatório, todos os pacientes utilizaram prednisona $40 \mathrm{mg} / \mathrm{dia}$, via oral, por aproximadamente 30 dias. Todos os pacientes utilizaram indefinidamente lente de contato terapêutica (LCT). Medicações tópicas incluíram acetato de prednisolona $1 \%$ colírio de $3 / 3$ horas nas primeiras semanas e após, conforme a necessidade, antibioticoprofilaxia contínua com fluorquinolona de $4^{\underline{a}}$ geração (gatifloxacina) $6 / 6$ horas, medroxiprogesterona $1 \%$ colírio $12 / 12$ horas e lágrimas artificiais sem conservantes à vontade. Dois pacientes com deficiência límbica grave (olhos 3 e 7) mantiveram-se usando soro autólogo.

Todos os olhos tiveram acompanhamento pós-operatório com acuidade visual com e sem correção, biomicroscopia, fundoscopia, campo de visão manual (CV) seriado a cada 3 meses e aferição da pressão intra-ocular (PIO) pela medida bidigital em todas as consultas. O tempo médio de seguimento foi de 11,2 meses (de 2 a 25 meses). Apenas 1 paciente (olho 4) perdeu o acompanhamento no $3^{\circ}$ mês, sendo esse tempo considerado para o seguimento.

Os olhos foram avaliados quanto a intercorrências pósoperatórias, comportamento campimétrico e resultado de acuidade visual final corrigida.

\section{RESULTADOS}

\section{Acuidade visual}

A AV corrigida pós-operatória foi melhor ou igual a 20/100 em $88,9 \%$ dos olhos (20/25 em 2 olhos, 20/30 em 1 olho, 20/40 em 1 olho, 20/50 em 2 olhos, 20/100 em 2 olhos) e movimento de mãos em 1 olho.

\section{Complicações pós-operatórias}

Sete dos 9 olhos $(77,8 \%)$ apresentaram alguma intercorrência no pós-operatório, sendo que em 3 olhos as intercorrências foram graves $(33,3 \%)$. Três olhos apresentaram opacidade de cápsula posterior, sendo dois tratados com capsulotomia por YAG laser no $3^{\circ}$ e $5^{\circ}$ mês pós-operatório, o terceiro caso (olho 7) está em acompanhamento; em três olhos houve formação de fibrina na câmara anterior, dois no $1^{\circ}$ - mês de pós-operatório e um no $4^{\circ}$ mês, dois sendo tratados com injeção de ativador do plasminogênio tecidual (tPA) na câmara anterior e um apenas com corticoterapia. Aumento da PIO (bidigital) foi identificado no 3 ํㅡês pós-operatório em 2 olhos, sendo controlado com medicação.

Em relação às complicações consideradas graves, dois olhos dos três envolvidos apresentavam quadros de importante deficiência límbica, a despeito dos transplantes de limbo feitos anteriormente. Um destes olhos evoluiu para um quadro de endoftalmite fúngica no $5^{\circ}$ mês pós-operatório, tratada com transplante de córnea a quente, explante da ceratoprótese e LIO, vitrectomia anterior, voriconazole intravítreo, intracameral, e sistêmico (endovenoso e oral), tendo sido este olho submetido a novo implante de ceratoprótese 2 meses após o tratamento da complicação. O diagnóstico da endoftalmite fúngica foi feito pela cultura do material vítreo (Candida albicans) e pela presença de esporos no anátomo-patológico. A patologia de base desta paciente era SSJ e a mesma já havia sido submetida à imunossupressão sistêmica por ocasião dos transplantes de córnea e limbo prévios e realizado pulsoterapia por papilite e edema macular cistóide após capsulotomia por YAG (no $3^{\circ}$ mês pós Kpro), além de ter apresentado no passado quadro de endoftamite fúngica no olho contralateral, que evoluiu para phthisis bulbi. O outro olho apresentou "melting" da córnea tectônica utilizada de suporte para a ceratoprótese, secundário a um defeito epitelial persistente, sendo necessária nova intervenção cirúrgica, com a troca do botão tectônico e novo transplante de limbo. O terceiro olho apresentou hipotonia por Seidel na interface das córneas doadora-receptora, que cedeu apenas após 6 intervenções cirúrgicas (Tabela 2).

Sete olhos mantiveram CV estáveis, de pelo menos $30^{\circ}$. Um olho ainda não tinha tido condições de realizar o exame (olho 6) e em outro, com glaucoma terminal e com acuidade visual de movimento de mãos, o exame não foi realizado.

\section{DISCUSS $\tilde{A} O$}

A literatura tem evidenciado bons resultados após implante da KPro de Dohlman-Doane em casos com pobre prognóstico para transplante convencional. Aquavella et al. relataram AV final melhor ou igual a 20/200 em $40 \%$ dos seus casos e melhor ou igual a 20/40 em 12\%, em até 2 anos de seguimento $^{(3)}$ e Dohlman, AV melhor ou igual a 20/100 em 47\% dos casos e melhor ou igual a 20/60 em 29\% dos seus $\operatorname{casos}^{(7)}$. Em nossa série de casos a AV final corrigida foi melhor ou igual a $20 / 100$ em $88,9 \%$ e em $44,4 \%$ dos casos melhor ou igual a 20/40 em um seguimento médio de 11 meses.

O erro refrativo variou na nossa série de casos entre $+9,5$ a -2,0 dioptrias esféricas e não excedeu a -2,0 dioptrias cilíndricas. Esta variação é semelhante à descrita na literatura ${ }^{(2)}$.

Em três casos $(33,3 \%)$ houve formação de membrana inflamatória na câmara anterior, apresentando boa evolução após injeção de tPA ou corticoterapia. A formação de membrana retro-protética é uma complicação descrita em $12 \%{ }^{(3)} \mathrm{a}$ $32 \%{ }^{(7)}$ dos casos na literatura.

Na nossa série de casos, ocorreu um caso de endoftalmite fúngica por Candida albicans em uma paciente cuja patologia de base era a síndrome de Stevens-Johnson, já descrita como fator de risco para endoftalmite bacteriana ${ }^{(8)}$ entre outras complicações ${ }^{(9)}$, além de apresentar história de imunossupressão prévia e endoftalmite fúngica no olho contralateral. O risco de endoftalmite fúngica na literatura é estimado em 0,009 casos por paciente/ano, sendo o uso de vancomicina como antibiótico profilático e o emprego de lente de contato terapêutica (LCT) fatores de risco importantes para o seu desenvolvimento ${ }^{(4)}$. Neste caso, a paciente utilizava LCT e fluoroquinolona 
como antibioticoprofilaxia. Dohlman relatou 3\% de ceratites micóticas em sua série de $\operatorname{casos}^{(7)}$.

$\mathrm{O}$ uso permanente da lente de contato terapêutica foi preconizado para retardar a desidratação e minimizar o efeito de "dellen" ocasionado pela face anterior da ceratoprótese, assim prevenindo a necrose e "melting" da córnea doadora ${ }^{(1)}$. Em nossa amostra, o "melting" corneano ocorreu em um paciente, mas aparentemente foi conseqüente de um defeito epitelial persistente secundário à deficiência límbica grave. Optamos pela troca da córnea e novos transplantes de limbo do pai e da mãe e, com esta medida, obtivemos epitelização e estabilização da superfície. Além disso, este paciente manteve-se em uso de soro autólogo e medroxiprogesterona, medidas que auxiliam na epitelização da superfície $^{(10)}$ e prevenção do "melting"(11), respectivamente. O re- cobrimento conjuntival sobre o botão doador é outra alternativa terapêutica $^{(1)}$ que pode ser utilizada. Em uma série de 25 casos da KPro, não foi encontrado "melting" em nenhum olho(2), já em uma série maior, de 63 olhos, necrose tecidual e "melting" ocorreram em 18 olhos $(29 \%)^{(12)}$. Mais recentemente, Dohlman relatou $1 \%$ de necrose corneana da interface no seguimento de 218 olhos $^{(7)}$.

O glaucoma é uma complicação freqüente e também ameaçadora do prognóstico visual em olhos tratados com KPro. Na maioria das vezes, a própria doença base é responsável pelo risco aumentado desta complicação ${ }^{(13)}$, além da opacidade e irregularidade corneanas prejudicarem o diagnóstico do glaucoma. Estudos encontraram uma prevalência de glaucoma de $64 \%$ nos 55 olhos tratados com KPro de Dohlman, sendo que 36\% tiveram diagnóstico previamente ao implante e $28 \%$ após a cirurgia ${ }^{(13)}$. Os

\begin{tabular}{|c|c|c|c|c|c|c|}
\hline Olho & $\begin{array}{l}\text { Procedimentos } \\
\text { adjuvantes }\end{array}$ & $\begin{array}{l}\text { Tipo } \\
\text { Kpro }\end{array}$ & $\begin{array}{l}\text { Intercorrências } \\
\text { (tratamento) }\end{array}$ & $\begin{array}{c}\mathrm{CV} \\
\text { manual }\end{array}$ & TS & $\begin{array}{l}\text { AV c/c pós-op } \\
\text { (meses) }\end{array}$ \\
\hline 1 & $\begin{array}{l}\text { Facectomia }+ \\
\text { LIO }\end{array}$ & PF & $\begin{array}{c}\text { OCP (YAG) } \\
\text { Simbléfaro (enxerto mucosa labial) }\end{array}$ & $30^{\circ}$ & 25 & $\begin{array}{c}20 / 40 \\
-1,5 \text { DE }-2,00 \text { DC } 90^{\circ}\end{array}$ \\
\hline 2 & $\begin{array}{c}\text { Facectomia }+ \\
\text { LIO + implante Ahmed }\end{array}$ & PF & Ausentes & $40^{\circ}$ & 23 & $\begin{array}{l}20 / 25 \\
+1,00 \mathrm{DE}\end{array}$ \\
\hline 3 & -- & PF & $\begin{array}{c}\text { Infiltrados corneanos (corticoterapia) } \\
\text { OCP (YAG) } \\
\text { Papilite + EMC (pulsoterapia) } \\
\text { Necrose corneana } \\
\text { Endoftalmite fúngica (Tx quente, } \\
\text { explante KPro/LIO, vitrec. ant.) } \\
\text { Reimplante Kpro } \\
\text { Neurite óptica (corticoterapia) }\end{array}$ & $\begin{array}{c}30^{\circ} \\
\text { aumento } \\
\text { da } \\
\text { mancha } \\
\text { cega }\end{array}$ & 19 & $\begin{array}{c}20 / 100 \\
+9,50 \mathrm{DE}\end{array}$ \\
\hline 4 & $\begin{array}{l}\text { Vitrectomia + } \\
\text { reposic. LIO }\end{array}$ & PF & $\uparrow$ PIO bidigital (acetazolamida) & $40^{\circ}$ & $3^{*}$ & $\begin{array}{c}20 / 100 \\
+5,0 \text { DE }-0,75 \text { DC } 75^{\circ}\end{array}$ \\
\hline 5 & $\begin{array}{l}\text { Facectomia } \\
\quad+\text { LIO }\end{array}$ & PF & Fibrina pré LIO (tPA) & $30^{\circ}$ & 12 & $\begin{array}{l}20 / 25 \\
-2,00 \mathrm{DE}\end{array}$ \\
\hline 6 & -- & Af & $\begin{array}{c}3 \text { episódios Seidel, inferior e tem-inf. } \\
\text { (Hystoacryl } \mathrm{I}^{\circledR} \text { ) } \\
\text { Mal posic. tubo Ahmed } \\
\text { (reposicionamento + TMA + Recob. } \\
\text { conj. c/ Beriplast }{ }^{\circledR} \text { P) } \\
\text { Necrose interface receptor-doador } \\
\text { inferior (Patch escleral + Recob. conj.) } \\
2 \text { episódios Seidel nasal-inferior } \\
\text { (Patch escleral + Recob. conj. c/ } \\
\text { Beriplast }{ }^{\circledR} \text { P) }\end{array}$ & -- & 7 & $\begin{aligned} & 20 / 50 \\
+ & 3,50 \mathrm{DE}\end{aligned}$ \\
\hline 7 & $\begin{array}{c}\text { Facectomia + } \\
\text { LIO + } \\
\text { reconstrução } \\
\text { superfície MA + } \\
\text { Tx limbo } \\
\text { alogênico }\end{array}$ & PF & $\begin{array}{c}\text { Fibrina pré LIO (tPA) } \\
\text { Defeito epit. persistente } \\
\text { (Tx limbo mãe) } \\
\text { "melting" corneano (troca da córnea } \\
\text { tectônica }+ \text { Tx limbo pai) } \\
\text { OCP (em acompanhamento) } \\
\uparrow \text { PIO bidigital } \rightarrow \text { maleato de timolol }\end{array}$ & $30^{\circ}$ & 6 & $\begin{array}{c}20 / 30 \\
-2,00 \mathrm{DE}\end{array}$ \\
\hline 8 & $\begin{array}{l}\text { Facectomia } \\
+ \text { LIO }\end{array}$ & PF & $\begin{array}{c}\text { EMC } \\
\text { Fibrina pré LIO (corticoterapia) }\end{array}$ & $30^{\circ}$ & 4 & $20 / 50$ \\
\hline 9 & $\begin{array}{l}\text { Vitrectomia } \\
\text { anterior }+ \\
\text { implante Ahmed }\end{array}$ & Af & Ausentes & -- & 2 & $\begin{array}{l}\text { MM } \\
\text { Escavação total } \\
\text { Atrofia retiniana }\end{array}$ \\
\hline \multicolumn{7}{|c|}{ 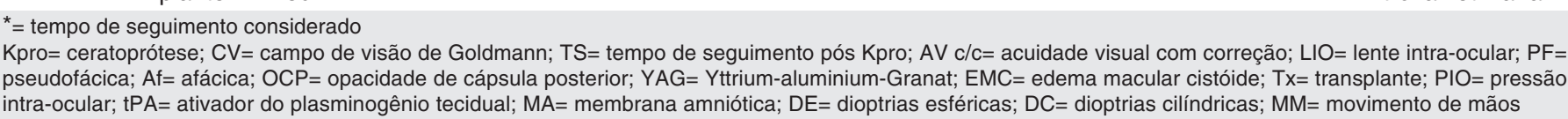 } \\
\hline
\end{tabular}


pacientes com diagnóstico já estabelecido foram submetidos ao implante de drenagem valvulado no mesmo tempo cirúrgico da KPro, necessitando do uso de medicação hipotensora em apenas $25 \%$ dos $\operatorname{casos}^{(13)}$. Dohlman relatou $51 \%$ de casos de glaucoma na sua amostra, com necessidade de implante valvulado de Ahmed em $80 \%$ deles $^{(7)}$. Em nossa amostra, dos 4 olhos com queimadura alcalina, 2 desenvolveram glaucoma, sendo um diagnosticado antes do implante da ceratoprótese (olho 2) e um após (olho 7). Pacientes com queimadura química grave parecem ser mais suscetíveis ao desenvolvimento do glaucoma ${ }^{(12)}$.

A dificuldade de avaliação do nervo óptico, por opacidade de meios, e da PIO, pelas alterações da superfície corneana, subestimam as taxas de glaucoma pré-operatório, comprometendo o prognóstico desses pacientes e obrigando, muitas vezes, a necessidade de nova cirurgia para implante de drenagem. Na nossa série de casos, 3 olhos tinham diagnóstico de glaucoma antes do tratamento com a KPro $(33,3 \%)$, um dos olhos já com o implante da válvula de Ahmed e os outros dois submetidos ao implante de drenagem simultaneamente à ceratoprótese. Estes olhos apresentaram bom controle pressórico com uso de hipotensor tópico no pós-operatório. Dois olhos apresentaram aumento da PIO após o implante da KPro, mas obtiveram bom controle com tratamento clínico. Todos os pacientes mantiveram CV seriados estáveis durante o período de seguimento.

O tempo de seguimento ainda é bastante limitado, mas nossa série inicial de casos sugere ser esta uma alternativa promissora para casos onde o transplante penetrante de córnea tem um prognóstico muito reservado, como falência de múltiplos transplantes prévios e doenças graves da superfície ocular. A principal vantagem da ceratoprótese de Dohlman-Doane é a ausência de necessidade de imunossupressão sistêmica aliada ao bom resultado visual. Casos de queimadura alcalina e de alto risco de rejeição, como múltiplos transplantes prévios apresentaram o melhor prognóstico em nossa série.

\section{ABSTRACT}

Purpose: To describe 9 eyes in 8 patients who received DohlmanDoane type 1 keratoprosthesis (KPro) with a mean follow-up of 11.2 months (2 to 25 months). Methods: A retrospective, noncomparative interventional case series. Previous corneal disease was alcaline burn in 4 eyes, multiple graft failure in 3 eyes, Stevens-Johnson syndrome in 1 eye and thermal injury in 1 eye. Best corrected visual acuity (BCVA) was hand motions or worse in all patients. Glaucoma was present preoperatively in
3 eyes and received Ahmed valve implantation. Results: 88,9\% eyes achieved BCVA of better than or equal to 20/100, and $44,4 \%$ better than or equal to $20 / 40$. In the postoperative period, 3 eyes developed posterior capsule opacity treated with YAG laser capsulotomy; 3 retroprosthetic membrane treated with tPA injection or steroids; 2 glaucoma in clinical treatment; 1 corneal melting, treated with donor cornea bottom exchange; and 1 fungic endophthalmitis, treated with corneal transplant, anterior vitrectomy, KPro and intraocular lens explantation, and specific intravitreal and endovenous treatment. Conclusion: Dohlman-Doane K-Pro seems to be a good option for cases of corneal blindness with poor prognosis for traditional penetrating keratoplasty. Its main advantage is not requesting systemic immunossuppression. Best results were achieved in non-immune diseases.

Keywords: Prostheses and implants/methods; Keratoplasty, penetrating; Prosthesis implantation/methods; Corneal diseases/surgery; Blindness; Case reports [publication type]

\section{REFERÊNCIAS}

1. Ma JJ, Graney JM, Dohlman CH. Repeat penetrating keratoplasty versus the Boston keratoprosthesis in graft failure. Int Ophthalmol Clin. 2005;45(4):49-59.

2. Aquavella JV, Qian Y, McCormick GJ, Palakuru JR. Keratoprosthesis: the Dohlman-Doane device. Am J Ophthalmol. 2005;140(6):1032-8.

3. Aquavella JV, Qian Y, McCormick GJ, Palakuru JR. Keratoprosthesis: current techniques. Cornea. 2006;25(6):656-62.

4. Barnes SD, Dohlman CH, Durand ML. Fungal colonization and infection in Boston keratoprosthesis. Cornea. 2007;26(1):9-15.

5. Doane MG, Dohlman CH, Bearse G. Fabrication of a keratoprosthesis. Cornea. 1996;15(2):179-84.

6. Dohlman CH, Waller SG, Netland PA. Keratoprosthesis surgery. In: Lindquist TD, Lindstrom RL, editors. Ophthalmic surgery update. $4^{\text {th }}$ ed. Chicago: Mosby-Year Book; 1997. p.1-31.

7. Dohlman CH. Results of 218 eyes with keratoprosthesis. ASCRS Meeting. San Diego, USA, 2007.

8. Dohlman CH, Barnes SD, Nouri M, et al. Preventing endophthalmitis in keratoprostheses: prophylactic antibiotic regimens. Invest Ophthalmol Vis Sci. 2003; 44:S108.

9. Dudenhoefer EJ, Nouri M, Gipson IK, Baratz KH, Tisdale AS, Dryja TP, et al. Histopathology of explanted collar button keratoprostheses: a clinicopathologic correlation. Cornea. 2003;22(5):424-8.

10. Tsubota K, Goto E, Shimmura S, Shimazaki J. Treatment of persistent corneal epithelial defect by autologous serum application. Ophthalmology. 1999;106(10):1984-9.

11. Hicks CR, Crawford GJ. Melting after keratoprosthesis implantation: the effects of medroxyprogesterone. Cornea. 2003;22(6):497-500.

12. Yaghouti F, Nouri M, Abad JC, Power WJ, Doane MG, Dohlman CH. Keratoprosthesis: preoperative prognostic categories. Cornea. 2001;20(1):19-23.

13. Netland PA, Terada H, Dohlman $\mathrm{CH}$. Glaucoma associated with keratoprosthesis. Ophthalmology. 1998;105(4):751-7. 